\title{
ACTUALIZACIONES
}

\section{Factores tróficos en neuronas dopaminérgicas mesencefálicas}

\author{
Pinzón G. ${ }^{1}$, Hurtado $\mathrm{H}^{2}{ }^{2}$
}

\begin{abstract}
Resumen
Desde su descubrimiento, se ha postulado, y en algunos casos comprobado, que los factores tróficos juegan un papel importante en el desarrollo, mantenimiento y regeneración del sistema nervioso. El conocimiento y la manipulación de estas sustancias ha servido para: a) formular nuevas teorías acerca de la etiología de varios desórdenes neurodegenerativos yb) desarrollarnuevas alternativas terapéuticas para estas entidades. En la presente revisión, resumimos los principales efectos de diferentes factores tróficos sobre las neuronas dopaminérgicas de la substantia nigraen desarrollo, cuya degeneración en el adulto ocasiona la mayoría de los síntomas observados en la enfermedad de Parkinson.
\end{abstract}

\section{Summary}

Since the discovery of trophic factors, their role in the development, maintenance and regeneration of the nervous system has been postulated and in some cases, proven. The knowledge and manipulation of these substances has been used: i) to formulate new theories about the etiology of various neurodegenerative disorders and ii) to develop new therapeutic alternatives in the treatment of these diseases. In this review, we summarize the main effects of several trophic factors on the developing dopaminergic neurons of the substantia nigra, whose degeneration causes, in the adult, most of the symptoms seen in Parkinson's disease.

Las grandes limitaciones del sistema nervioso central (SNC) de los primates adultos en cuanto a su capacidad para formar nuevas neuronas a partir de precursores inmaduros (1) y la idea generalizada de que se requiere ungrupo neuronal estable para retener el aprendizaje adquirido, hicieron pensar durante mucho tiempo que era imposible una regeneración funcional de neuronas y redes neuronales lesionadas en el SNC adulto (2). Sin embargo, durante los últimos años se ha demostrado que el sistema nervioso no permanece estático y que, por el contrario, posee mecanismos adaptativos que tratan de restaurar

1 Médico general, División de Investigación Básica, Instituto Nacional de Salud, Santafé de Bogotá, Colombia.

2 Biólogo Ph.D., División de Investigación Básica, Instituto Nacional de Salud, Santafé de Bogotá, Colombia. la función hacia un límite normal $(3,4)$. Estas observaciones han servido como base para desarrollar diferentes estrategias cuyo objetivo es promover la conservación y reparación del SNC (tabla 1). Estas estrategias involucran la identificación y estudio de señales ambientales que afectan la supervivencia y maduración neuronal, así como la especificidad de sus blancos de inervación durante estadíos críticos del desarrollo (5). Es así como en la enfermedad de Parkinson, uno de los síndromes neurodegenerativos más comunes (6), la investigación se ha dirigido al reconocimiento y análisis de los efectos que este tipo de sustancias, conocidas como factores tróficos, ejercen sobre el desarrollo de las células dopaminérgicas del mesencéfalo, creando nuevas perspectivas acerca de su uso, ya sea como coadyuvantes en la integración de 
Tabla 1. Estrategias para promover la conservación y/o reparación del SNC.

1. Trasplantes celulares $(80)$

a. Células de cerebro fetal

b. Células tumorales

c. Paraneuronas

d. Células modificadas por ingeniería genética

2. Injertos de siste:?a nervioso periférico en sistema nervioso central (81)

3. Guías sintéticas biocompatibles: proveen un soporte mecánico para dirigir fibras en crecimiento (5)

4. Utilización de bombas de liberación de fármacos, neurotransmisores y/o factores de crecimiento (82)

los implantes celulares $(7,8)$, o como factores que directamente impidan o al menos retarden la degeneración y muerte celular que subyacen esta enfermedad $(5,9)$. El estudio de estas señales es igualmente importante en la búsqueda de la etiología de la enfermedad de Parkinson, aún sin establecer (10-12), y para la cual se ha sugerido una deficiencia trófica durante el desarrollo de las neuronas dopaminérgicas $(13,14)$.

\section{Factores tróficos}

El desarrollo embrionario del sistema nervioso de los vertebrados puede ser visto como una serie de eventos secuenciales considerados tanto progresivos como regresivos. Los fenómenos progresivos incluyen la génesis de células neuronales, su determinación, proliferación, diferenciación y laformación de complejos patrones de conexiones o sinapsis que caracterizan el sistema nervioso maduro y funcional (15). Dentro delos eventos regresivos se destacan la eliminación de procesos celulares (axones) y la muerte neuronal (16-19). Estos fenómenos dependen de la interacción entre los miles de genes que se expresan en el sistema nervioso y una serie de señales medioambientales (humorales y celulares) particulares a cada tipo celular (20). La dinámica de esta interdependencia se cree que es regulada en gran parte por un grupo de polipéptidos denominados factores neurotróficos, los cuales son sintetizados por las células postsinápticas o blancos, células presinápticas y glia $(14,16,21,22)$. Estos factores son internalizados por procesos axonales y dendríticos, para posteriormente ser transportados retrógra- damente y/o anterógradamente al cuerpo de la neurona presináptica/postsináptica. Allí operan sobre la maquinaria metabólica celular, a través de la regulación de la transcripción génica, interviniendo de esta manera en el desarrollo, diferenciación y supervivencia neuronal $(21,23)$. En algunos casos se ha demostrado que las dependencias tróficas son obligatorias y que las neuronas a través de sus terminales nerviosas compiten unas con otras por diversos soportes regulatorios (19). Esta competencia parece ser la principal forma de establecer contactos sinápticos adecuados y de regular el número de neuronas y los grados de convergencia y divergencia de las vías neurales (24). La acción de los factores tróficos no se limita únicamente a las células del SNC en desarrollo; en las neuronas maduras desempeñan un papel importantísimo en el mantenimiento de la sinapsis, así como en el reestablecimiento $\mathrm{y} / \mathrm{o}$ desarrollo de nuevas conexiones después de una lesión $(3,25)$.

\section{Principales técnicas y procedimientos utili- zados en el estudio del desarrollo de las célu- las dopaminérgicas del sistema mesoestriatal}

Se han utilizado cultivos de explantes y de células disociadas para estudiar las características morfológicas, bioquímicas y fisiológicas de células dopaminérgicas provenientes del SNC de embriones y adultos de diferentes edades y distintas especies. Se han investigado sus interacciones con otras neuronas y células gliales, así como la manera en que estas células, factores tróficos y otros componentes de su microambiente regulan su desarrollo, diferenciación, supervivencia y especificidad de inervación de sistemas funcionalmente activos.

La mayoría de los estudios y resultados presentados a continuación han utilizado sistemas in vitro, en los cuales la influencia de los factores tróficos ha sido evaluada observando principalmente la división y/o supervivencia neuronal, el crecimiento de neuritas y la diferenciación bioquímica. El análisis de estos efectos se ha realizado empleando técnicas inmunohistoquímicas y de histofluorescencia, a través del uso de anticuerpos que reconocen a la dopamina y a la tirosina hidroxilasa $(\mathrm{TH})$, la enzima limitante en la 
rata de síntesis de catecolaminas. El secuenciamiento del mRNA que codifica para la síntesis de la $\mathrm{TH}(26)$, ha permitido la realización de diferentes estudios, principalmente de expresión génica, por medio de la hibridización in situ(27). También se ha empleado, como marcador de diferenciación, la captación de la ${ }^{3} \mathrm{H}$-dopamina y de su síntesis a partir de la ${ }^{3} \mathrm{H}$-tirosina, y otros procedimientos dirigidos a la determinación de metabolitos de la dopamina y a la medición de la actividad de enzimas como la catecol-O-metiltransferasa (COMT) y la monoamino-oxidasa (MAO), responsables de la inactivación catabólica de las catecolaminas.

Muchos de los trabajos experimentales se han realizado utilizando células dopaminérgicas obtenidas a partir de embriones de ratón de 13-15 días de edad (E13 a 15) y rata (E14-16), las cuales aún no han inervado su blanco estriatal. Pocos estudios se han hecho en embriones de menor edad, debido a que el número de células $\mathrm{TH}+$ es muy bajo (28) y a que la disección del mesencéfalo ventral en embriones menores de 13 días es bastante difícil.

La caracterización y análisis de las células dopaminérgicas, cultivadas en un medio sin suero, ha revelado dos tipos morfológicos, que semejan notablemente aquéllos observados in vivo: células fusiformes y células multipolares. Estas últimas tienen forma piramidal, son altamente fluorescentes (autofluorescencia de catecolaminas en presencia de vapores de formaldehído) y exhiben pálidos procesos de longitud variable, frecuentemente terminados por un cono de crecimiento. Las células fusiformes presentan dos largos procesos opuestos, que se ramifican a cierta distancia de el cuerpo celular. Los cultivos se han seguido hasta por seis semanas; las células incrementan gradualmente su tamaño, hasta alcanzar un aspecto maduro al finalizar este período (29).

Por último, luego de demostrarse un efecto positivo sobre el desarrollo y/o mantenimiento de las neuronas dopaminérgicas, se ha estudiado si algunos factores tróficos pueden proteger estas células contra neurotoxinas tales como la 6OHDA ( 6-hidroxidopamina) y el MPP+ (1-metil4-fenilpiridina). La 6-OHDA y el MPP+ producen degeneración de las neuronas dopaminérgicas de la vía nigro-estriatal (30-32), afectada en la enfermedad de Parkinson. El uso de neurotoxinas ha permitido crear modelos animales de esta enfermedad $(30,32,33)$ los cuales han servido para estudiar su etiología (34) y fisiopatología (35), así como para desarrollar nuevas alternativas terapéuticas (36).

\section{Desarrollo}

Las células dopaminérgicas de la substantia nigra y el área ventral tegmental surgen del istmo de la fóvea y del istmo del romboencéfalo (28), para llegar a ser la principal fuente de inervación dopaminérgica del cuerpo estriado y la corteza frontal. Las neuronas dopaminérgicas migran desde la zona ventricular a lo largo del acueducto de Silvio hasta la superficieventral delmesencéfalo en desarrollo. Esta migración se realiza en forma radial, mediante un patrón de ensamblaje conocido como inside-out, característico de estructuras laminares, en el cual las neuronas generadas más tardíamente migran tomando sucesivamenteposiciones exteriores con respectoa las neuronas ya formadas (37), en un proceso que parece ser dirigido por la glía radial (38).

La ontogenia de las células dopaminérgicas ha sido investigada en varias especies, principalmente en ratas (39) y ratones. En estos últimos, Di Porzio et al. (28), encontraron células $\mathrm{TH}+$ en el día E8,5-9, mientras aparentemente aún estaban migrando desde la capa proliferativa. Esto sugiere que en el caso de esta vía, la producción de un neurotransmisor característico puede ocurrir antes que las neuronas alcancen su posición final. El número de células catecolaminérgicas aumenta en los días siguientes, observándose hacia el día E10,5-11 células con neuritas $\mathrm{TH}+$, cuya distribución recuerda los grupos A9 y A10 del ratón adulto. Sin embargo, sólo hasta el día E13 se aprecian células $\mathrm{TH}+$ como dos grupos independientes (28). En los días E13 y E14 se ha reportado un incremento de 15 veces en la cantidad de células $\mathrm{TH}+$, el cual parece representar un pico mitótico. Entre E14 y E18 el incremento es mínimo (40). La concentración de dopamina se correlaciona con el aumento en el número de células $\mathrm{TH}+$ y con el comienzo de la inervación estriatal por parte de estas células (40). Lo ante- 
rior parece reflejar un estímulo en la síntesis de dopamina por parte de las células estriatales blanco.

Los mecanismos de recaptación de dopamina aparecen en una edad gestacional relativamente tardía, aproximadamente E16, coincidiendo nuevamente con la llegada de fibras dopaminérgicas al estriado (40). Estos resultados señalan que la maduración de lasneuronas dopaminérgicas se relaciona, al menos parcialmente, con el desarrollo de las células blanco (40). In vitro, el estudio del efecto regulatorio de las células blanco sobre el desarrollo morfológico y bioquímico de las neuronas aferentes dopaminérgicas hallevado a la misma conclusión. Prochiantz et al. (41) y Daguet etal. (42), demostraron, utilizando células mesencefálicas de ratón (E13-15), que los cocultivos con células estriatales (E15) facilitan el desarrollo, maduración y supervivencia de las células dopaminérgicas, al aumentar los sitios de captación y síntesis de dopamina. Esta interacción no parece influir sobre el fenómeno de liberación de dopamina por parte de los procesos neuronales (42). Sin embargo, la presencia, tipo y distribución de estos procesos, así como sus relaciones morfológicas son dependientes del cocultivo con células blanco apropiadas, sugiriendo que la información necesaria para esta diferenciación es en gran parte intrínseca a las neuronas dopaminérgicas y a sus células blanco (43).

La regulación promovida por las células estriatales parece ser mediada por un factor proteico ligado a la membrana de las células estriatales (44). Además, también parece intervenir un péptido difusible de origen estriatal, responsable en parte de la proliferación y mantenimiento de los axones dopaminérgicos en diferentes estadíos del desarrollo pre y postnatal (45). Del Toso et al. (46), encontraron que la actividad de un factor difusible al que denominaron factor neurotrófico derivado del estriado(SDNF) está asociada con una proteína básica de un peso molecular aproximado de 14 kD. Aunque este tipo de experimentos no permitió identificar con certeza los factores responsables de la supervivencia y maduración de las células dopaminérgicas, sí abrieron la puerta a los ensa- yos con factores ya conocidos por su efecto en otros sistemas.

\section{Efecto de factores tróficos definidos sobre las células mesencefálicas}

Diferentes grupos de investigadores han trabajado con sustancias previamente reconocidas por sus influencias sobre tejidos neuronales y no neuronales, tales como los factores de crecimiento fibroblástico básico y ácido (bFGF, aFGF), insulina, factores de crecimiento similares a la insulina I y II (IGF-I, IGF-II), factor de crecimiento epidérmico (EGF), factor de crecimiento derivado de plaquetas (PDGF), factor de crecimiento transformante $a$, factor de crecimiento derivado de cerebro (BDNF) y el factor de crecimiento nervioso (NGF). Como resultado de estos estudios se ha demostrado que los factores anteriormente anotados intervienen con diversos grados de selectividad sobre el desarrollo de neuronas dopaminérgicas centrales (tabla 2).

El bFGF es un polipéptido que se halla ampliamente distribuído en el cerebro (47) y cuya

Tabla 2. Efecto de factores tróficos definidos sobre la supervivencia celular,crecimiento de neuritas y diferenciación bioquímica de células dopaminérgicas embrionarias.

\begin{tabular}{lllll}
\hline $\begin{array}{l}\text { Factor } \\
\text { trófico }\end{array}$ & $\begin{array}{c}\text { Supervivencia } \\
\text { celular }\end{array}$ & $\begin{array}{c}\text { Crecimiento de } \\
\text { neuritas }\end{array}$ & $\begin{array}{c}\text { Diferenciación } \\
\text { bioquimica }\end{array}$ \\
\hline bFGF & $+(49)$ & $+(49)$ & $+(49-52)$ \\
aFGF &. & $+(55)$ & $+(55)$ \\
IGF-I &. & $\cdot$ & $+(51)$ \\
IGF-II &. &. & $+(51)$ \\
Insulina &. & csss & $+(51)$ \\
PDGF-BB & $+(58)$ & s & + & \\
EGF & $+(51,52)$ & $+(51,52)$ & $+(51,52)$ \\
TGFa & $+(59)$ & $+(59)$ & $+(59)$ \\
BDNF & $+(62)$ & $+(61)$ & $+(60,61)$ \\
NGF & $-(51)$ & $-(51)$ & - & $(51)$ \\
\hline
\end{tabular}

+ : efecto positivo.

-: efecto negativo

$\therefore$ no se ha reportado.

Los números entre paréntesis corresponden a las referencias de los efectos citados. 
presencia ha sido demostrada en el mesencéfalo ventral, a partir de E16 (48), lo que sugiere un papel in vivo de este factor sobre el desarrollo de las células dopaminérgicas. Por otra parte, son contradictorios los reportes acerca del efecto del bFGF sobre el desarrollo de células mesencefálicas in vitro; Ferrari etal. (49), utilizando cultivos de células mesencéfalicas disociadas de rata (E14), en los cuales la gran mayoría (>99\%) de las células teñían positivamente con anticuerpo antineurofilamento, encontraron que el bFGF aumenta el número de células sobre vivientes así como el grado de ramificación de los procesos.

Hartikka, Staufenbiel y Lubbert (50) no observaron ningun efecto significativo del bFGF sobre la supervivencia de células dopaminérgicas provenientes de ratones de la misma edad. Cabe anotar, que los cultivos de estos investiga-dores no fueron tan puros, ya que encontraron gran cantidad de astrocitos (células marcadas positivamente con anticuerpos antiproteína ácida fibrilar glial). Estas diferencias son atribuidas a las condiciones de cultivo. Sin embargo, varios grupos han reportado un aumento en la captación de dopamina bajo la influencia del bFGF, lo que sugiere un efecto principal sobre la diferenciación (49-52).

Park y Miytilineou (52) encontraron que el bFGF también estimula la proliferación de astrocitos y que su acción sobre las neuronas dopaminérgicas coincide con un incremento en la densidad glial, sugiriendo que la proliferación glial media sus efectos tróficos. En un modelo animal de la enfermedad de Parkinson, el bFGF disminuyó los déficits ocasionados por la administración de la neurotoxina MPTP (1-metil-4-fenil-1,2,3,6tetrahidroxipiridina, cuya oxidación produce MPP+) (53); si a esto sumamos la reciente comunicación acerca de la disminución en la concentración del bFGF en neuronas de la substantia nigra obtenida de muestras postmortem de individuos con diagnóstico de enfermedad de Parkinson (54), parece sugestivo el potencial del bFGF en el tratamiento de esta entidad. Sin embargo, su capacidad de estimular la proliferación de otros tipos celulares comolos astrocitos, podría complicar su eventual utilidad terapéutica (9).
Se ha reportado la presencia del mRNA del aFGF en las neuronas de la pars compacta de la substantia nigra en desarrollo. Lo anterior se ha correlacionado con la diferenciación, migración y establecimiento de sinapsis (55).

El EGF, polipéptido que afecta diferentes poblaciones celulares en el cerebro (56), produce variables incrementos en la captación de dopamina, supervivencia neuronal y crecimiento de neuritas $(51,53)$.

EL IGF-I y el IGF-II, polipéptidos con homología estructural a la proinsulina y que ejercen efectos metabólicos similares a la insulina (57), estimulan en las neuronas dopaminérgicas la expresión de sus neurotransmisores característicos (51). Esta diferenciación bioquímica fue igualmente promovida por la insulina (51).

EIPDGF es un factor que presenta tre isoformas: $A A, B B$ y CC (58). La isoforma PDGF-BB disminuye la pérdida celular progresiva en cultivos de células mesencefálicas de embriones de ratas (E14) y humanos, siendo este efecto específico para las células $\mathrm{TH}+$. La isoforma PDGF-AA no influye en la supervivencia de estas células. Estos resultados implican a la isoforma PDGF$\mathrm{BB}$ como un factor neurotrópico para las células dopaminérgicas en cultivo (58).

EI TGF a, in vitro, aumenta la captación de dopamina de manera dosis dependiente, incrementa selectivamente la supervivencia de células $\mathrm{TH}+\mathrm{y}$ aumenta el número y longitud de las ramificaciones, duplicando así el área de influencia de estas células (59).

EI BDNF, provoca varias respuestas importantes en cultivos de células mesencefálicas de embriones de rata (E14-16):

1-Duplica la captación de dopamina por parte de las neuronas dopaminérgicas luego de cuatro días de tratamiento $(60,61)$.

2- Disminuye la pérdida de células $\mathrm{TH}+$ después de ocho días de cultivo, siendo este efecto más pronunciado cuanto más temprano haya sido adicionado el BDNF a los cultivos (62).

3- Estimula la formación de neuritas primarias (61). 
Estos resultados sugieren que el BDNF promueve la supervivencia y/o diferenciación de neuronas dopaminérgicas en cultivo. Más aún, elBDNF protege in vitro a estas neuronas contra el daño producido por las neurotoxinas 6-OHDA y MPP+ $(62,63)$. Al parecer esta protección se hace mediante un aumento en la actividad de la enzima glutatión reductasa, la cual impediría la oxidación del glutatión que ha sido asociada a la toxicidad provocada por la 6-OHDA (64). Sin embargo, el BDNF no aumenta la supervivencia de injertos dopaminérgicos al estriado de ratones lesionados con 6-OHDA; su efecto parece limitarse a aumentar la capacidad funcional de las células transplantadas (65). Hyman et al. (62), consideran posible que el BDNF sea el mismo factor difusible reportado por Del Toso et al. (46) como SDFN, quizás por la similitud de sus pesos moleculares y por el hallazgo del mRNA del BDNF en el estriado (66). Recientemente han encontrado mRNA de este factor expresándose en neuronas del área tegmental ventral y la substantia nigra en ratas adultas (67), lo que sugiere un soporte trófico local, hipótesis que se refuerza por la ausencia de este factor en el estriado (68). Gall et al. (69), consideran que la arquitectura de las conexiones entre las células dopaminérgicas del cerebro medio es compatible con el concepto de una liberación autocrina o paracrina del BDNF. Esto no es necesariamente incompatible con el modelo de soporte trófico derivado de la célula blanco.

EI NGF, el factor trófico mejor estudiado $(70,71)$, es reconocido por su influencia sobre el desarroIlo, crecimiento y diferenciación de las neuronas colinérgicas del cerebro anterior (72). Durante el desarrollo, su distribución y la de su receptor en el cerebro se extienden a otros tipos celulares $(73,74)$. Sin embargo, parece no tener ningún efecto sobre las células mesencefálicas en desarrollo (51).

\section{Papel de la glía en el desarrollo y diferencia- ción de las neuronas dopaminérgicas}

El efecto de la interacción glía-neurona durante el proceso de neurogénesis se relaciona con:

1. El papel que cumplen las fibras de la glía radial como guías de las neuronas migratorias (75).
2. Las interacciones tróficas entre astrocitos inmaduros y neuronas en desarrollo (22).

3. La organización que presentan algunas estructuras cerebrales como la corteza (76).

El efecto de esta interacción trófica sobre el desarrollo de las células dopaminérgicas aún no se ha precisado por completo. Di Porzio et al. (77), realizaron cocultivos de células mesencefálicas y células estriatales en medio libre de suero, sistema en el cual se obtienen poblaciones neuronales virtualmente puras. Estos investigadores encontraron que la reducción de la población glial no afecta la maduración de las neuronasy noaltera la respuesta de las células estriatales. Por el contrario, otros investigadores (78), usando una metodología en la cual la morfología individual de las células dopaminérgicas puede ser estudiada luego de la captación de ${ }^{3} \mathrm{H}$ dopamina, reportaron que las células gliales ejercen un efecto morfogenético sobre las neuronas dopaminérgicas, consistente en que al crecer sobre una monocapa de glia mesencefálica, estas neuronas adquieren un patrón morfológico que coincide con las geometría de las células dopaminérgicas mesoestriatales in vivo. Estos resultados fueron confirmados por Beyer et al. (79) quienes utilizando cocultivos de glía mesencefálica de rata (E21) con células de la substantia nigra (E14) o neoestriado (E14), encontraron que la glía mesencefálica induce el desarrollo y promueve la supervivencia de células con propiedades dopaminérgicas $(\mathrm{TH}+)$ en cultivos de ambos orígenes, siendo mayor la diferenciación morfológica y la expresión de la $\mathrm{TH}$ en los cocultivos de glía y neuronas provenientes de la substantia nigra. Este efecto señala el probable papel inductor de la glía mesencefálica sobre neuronas o neuroblastos competentes para la diferenciación hacia un fenotipo dopaminérgico.

\section{Conclusiones}

Existen varios desórdenes neurodegenerativos en los cuales a pesar de que se encuentra una estrecha asociación entre ciertos grupos neuronales afectados y la sintomatología observada, no ha sido posible establecer aún su etiología ni mucho menos terapias que impidan o reviertan los procesos que conducen a la aparición 
de estas enfermedades $(9,13)$. La identificación de los factores tróficos como moléculas indispensables para el desarrollo, mantenimiento y reparación del SNC, ha generado muchas expectativas sobre su papel etiológico y potencial terapéutico. Debido a que en la enfermedad de Parkinson se ha demostrado la relación más clara entre los cambios patológicos, las alteraciones bioquímicas y los síntomas clínicos, es evidente la importancia que tiene el estudio del desarrollo de las células dopaminérgicas de la substantia nigra.

A pesar de toda la investigación realizada, aún permanecen muchos interrogantes por resolver:

1- Los receptores y las vías de transducción de las señales utilizadas por las células dopaminérgicas como respuesta a los factores tróficos. Un mejor conocimiento de la estructura tridimensional, subtipos e interacciones de los receptores con sus ligandos, permitiría realizar investigaciones que conduzcan a la búsqueday/o diseño de moléculas que puedan tener una utilidad terapéutica.

2- El papel de estos factores in vivo desde el punto de vista tanto fisiológico como terapéutico.

3- Los períodos específicos en el desarrollo en los cuales la actividad de los factores tróficos es más importante.

4- Los mecanismos de regulación de la expresión de estos factores.

5- Las interacciones que se puedan presentar entre ellos.

6- Identificar factores que determinen lineajes dopaminérgicos.

\section{Referencias}

1. Rakic P. Limits of neurogenesis in primates. Science 1985; 227:1054.

2. FawcettJW. Factors responsible for the failure of structural repair in the central nervous system. Chapter 6 . In: Hunter AJ, Clark M, eds. Neurodegeneration. Academic Press Inc. 1992:81.

3. Cotman CW, Nieto-Sampedro M. Cell biology of synaptic plasticity. Science 1984; 225:1287.

4. Waxman SG. Functional recovery in diseases of the nervous system. Advan Neurol 1988; 47:1.
5. Lipton SA. Growth factors for neuronal survival and process regeneration. Implications in the mammalian central nervous system. Arch Neurol 1989; 46:1248.

6. Kurtzke JF. Neuroepidemiology. Ann Neurol 1984; 16:265.

7. Nieto-Sampedro M, Kesslak JP, Gibbs R, et al. Effects of conditioning lesions on transplant survival, connectivity and function. Role of neurotrophic factors. Ann N Y Acad Sci 1987; 495:108.

8. Lindvali 0 . Prospects of transplantation in human neurodegenerative diseases. Trends Neurosci 1991; $14: 376$.

9. Eide FF, Lowenstein DH, Reichart LF. Neurotrophins and their receptors current concepts and implications for neurologic disease. Exp Neurol 1993; 121:200.

10. Marsden CD. Parkinson's disease. Lancet 1990; 1:1457.

11. Stern M, Dulaney E, Gruber S. The epidemiology of Parkinson's disease. A case-control study of youngonset and old-onset patients. Arch Neurol 1991; 48:903.

12. Semchuk KM, Love EJ, Lee RG. Parkinson's disease and exposure to agricultural work and pesticide chemicals. Neurology 1992; 42:1238.

13. Appel SH. A unifying hypothesis for the cause of amyotrophic lateral sclerosis, Parkinsonism and Alzheimer disease. Ann Neurol 1981; 10:499.

14. Snider WD, Johnson EMJ. Neurotrhopic molecules. Ann Neurol 1989; 26:489.

15. Sheperd GM. Developmental neurobiology. Chapter 9. In: Sheperd GM, Ed. Neurobiology, second ed. Oxford University Press 1988; 177.

16. Cunningham TJ. Naturally ocurring neuron death and its regulation by developing neural pathways. Int Rev Cyt 1982; 74:163.

17. Cowan WM, Fawcett JW, O'Leary DDM, et al. Regressive events in neurogenesis. Science 1984; 225:1258.

18. Clarke PGH. Neuronal death in the development of the vertebrate nervous system. Trends Neurosci 1985; 8:345.

19. Oppenheim RW. Cell death during development of the nervous system. Annu Rev Neurosci 1991; 14:453.

20. Sutcliffe JG, Milner RG, Gottesfeld JM, et al. Control of neuronal gene expression. Science 1984; 225:1308.

21. Varon S. Factors promoting the growth of the nervous system. Disscussions Neurosci 1985; 2:1.

22. Varon S, Manthorpe M, Davis GE. Growth factors. Advan Neurol 1988; 47:493.

23. Walicke PA. Novel neurotrophic factors and oncogenes. Ann Rev Neurosci 1989; 12:103.

24. Purves D, Lichtman JW. Neuronal death during development. Chapter 6. In: Purves D, Lichtman JW, eds. Principles of neural development. Sinauer Associates Inc. 1985:131.

25. Detta A, Grabham P, Hitchcok E. Phenotypic plasticity of mature human fetal mesencephalic dopaminergic neurons and glial cells. Restor Neurol Neurosci 1992; 4:41. 
26. Grima B, Lamouroux A, Blanot F, et al. Complete coding sequence of rat tyrosine hydroxylase mRNA. Proc Natl Acad Sci USA 1985; 82:21617.

27. Young WS, Bonner TI, Bann MR. Mesencephalic dopamine neurons regulate the expression of neuropeptide mRNAs in the rat forebrain. Proc Natl Acad Sci USA 1986; 83:9827.

28. Di Porzio U, Zuddas A, Cosenza-Murphy DB, et al. Early appearence of tyrosine hydroxilase immunoreactive cells in the mesencephalon of mouse embryos. Int J Dev Neurosci 1990; 8:523.

29. Berger B, Di Porzio U, Daguet M-C, et al. Long-term development of mesencephalic dopaminergic neurons of mouse embryos in dissociated primary cultures: morphological and histochemical characteristics. Neuroscience 1982; 7:193.

30. Burns R, Chiueh C, Markey S, et al. A primate model of Parkinsonism: selective destruction of dopaminergic neurons in the pars compacta of the substantia nigra by N-Methyl-4-Phenyl-1,2,3,6-Tetrahidropyridine. Proc Natl Acad Sci USA 1983; 80:4545.

31. Langston J, Ballard P, Tetrud J, et al. Chronic Parkinsonism in humans due to a product of meperidine analog synthesis. Science 1983; 219:977.

32. Casas M, Ferre S, Cobos A, et al. Comparison between apomorphin and amphetamine-induced rotational behaviour in rats with unilateral nigrostriastal pathway lesion. Neuropharmacology 1988; 27:657.

33. Chiueh CC, Burns RS, Markey SP, et al. Primate model of Parkinsonism: selective lesion of nigrostriatal neurons by 1-methyl-4-phenyl-1,2,3,6-tetrahydropyridine produces an extrapyramidal syndrome in rhesus monkeys. Life Sci 1985; 36:213.

34. Ikeda H, Markey C, Markey SP. Search for neurotoxins structurally related to 1-methyl-4-phenylpyridine (MPP+) in the pathogenesis of Parkinson's disease. Brain Res 1992; 575:285.

35. Irwin I, DeLanney LE, Forno LS, et al. The evolution of nigrostriatal neurochemical changes in the MPTP-treated squirrel monkey. Brain Res 1990; 531:242.

36. Brundin P, Isacson O, Gage FH, et al. The rotating 6hydroxydopamine-lesioned mouse as a model for assessing functional effects of neuronal grafting. Brain Res 1986; 366:346.

37. Jacobson M. Histogenesis and morphogenesis of cortical structures. Chapter 10. In: Jacobson M, ed. Developmental neurobiology, third ed. Plenum Press 1991; 401.

38. Shults CW, Hashimoto R, Brady RM, etal. Dopaminergic cells align along radial glia in the developing mesencephalon of the rat. Neuroscience 1990; 38:427.

39. Voorn P, Kalsbeek A, Jorritsma-Byham B, et al. The pre and postnatal development of the dopaminergic cell groups in the ventralmesencephalon and the dopaminergic innervation of the striatum of the rat. Neuroscience 1988; 25:857.

40. Fiszman M, Zuddas A, Masana M, et al. Dopamine synthesis precedes dopamine uptake in embryonic rat mesencephalic neurons. J Neurochem 1991; 56:392.
41. Prochiantz A, Di Porzio U, Kato A, et al. In vitro maturation of mesencephalic dopaminergic neurons from mouse embryos is enhanced in presence of their striatal target cells. Proc Natl Acad Sci USA 1979; 76:5387.

42. Daguet M-C, Di Porzio U, Kato A, et al. Release of dopamine from dissociated mesencephalic dopaminergic neurons in primary cultures in abscence or presence of striatal target cells. Brain Res 1980; 191:254.

43. Hemmendinger LM, Garber BB, Hoffman PC, et al. Target neuron-specfic process formation by embryonic mesencephalic dopamine neurons in vitro. Proc Natl Acad Sci USA 1981; 78:1264.

44. Prochiantz A, Daguet M-C, Herbet A, et al. Specific stimulation of in vitro maturation of mesencephalic dopaminergic neurons by striatal membranes. Nature 1981; 293:570.

45. Tomozawa Y, AppelSH. Soluble striatal extracts enhance development of mesencephalic dopaminergic neurons in vitro. Brain Res 1986; 399:111.

46. Del Toso R, Giorgi O, Soranzo C, et al. Development and survival of neurons in dissociated fetal mesencephalic serum-free cell cultures: I. Effects of cell density and of an adult mammalian striatal-derived neuronotrophic factor (SDNF). J Neurosci 1988; 8:733.

47. Bean AJ, Elde R, Cao Y, et al. Expression of acidic and basic fibroblast growth factors in the substantia nigra of rat, monkey and human. Proc Natl Acad Sci USA 1991; 88:10237.

48. Bean AJ, Oelling C, Pettersson RF, et al. Differential expression of acidic and basic FGF in the rat substantia nigra during development. Neuroreport 1992; 3:993.

49. Ferrari G, Minozzi MC, Toffano AL, Skaper SD. Basic fibroblastic growth factor affects the survival and development of mesencephalic neurons in culture. In: Lauder JM, ed. Molecular aspects of development and aging of the nervous system. New York: Plenum Press 1990; 93.

50. Hartikka J, Staufenbiel M, Lubbert H. Cyclic AMP, but not basic FGF, increases the in vitro survival of mesencephalic dopaminergic neurons and protects them from MPP+ -induced degeneration. J Neurosci Res 1992; 32:190.

51. Knusel B, Michel PP, Schwaber JS, Hefti F. Selective and nonselective stimulation of central cholinergic and dopaminergic development in vitro by nerve growth factor, basic fibroblast growth factor, epidermal growth factor, insulin and the insulin-like growth factors. J Neurosci 1990; 10:558.

52. Park TH, Mytilineu C. Protection from 1-methyl-4phenylpyridinium $(\mathrm{MPP}+)$ toxicity and stimulation of regrowth of $\mathrm{MPP}(+)$-damaged dopaminergic fibers by treatment of mesencephalic cultures with EGF and basic FGF. Brain Res 1992; 599:83.

53. Otto D, Unsicker K. Basic FGF reverses chemical and morphological deficits in the nigrostriatal system ofMPTPtreated mice. J Neurosci 1990; 10:1912.

54. Tooyama I,Kawamata T, Walker D, et al. Loss of fibroblast growth factor in substantia nigra neurons in Parkinson's disease. Neurology 1993; 43:372. 
55. Wilcox BJ, Unnerstall JR. Expression of acidic fibroblast growth factor mRNA in the developing and adult rat brain. Neuron 1991; 6:397.

56. Mazzoni IE, Kenigsberg RL. Effects of epidermal growth factor in the mammalian central nervous system: its possible implications in brain pathologies and therapeutic applications. Drug Develop Res 1992; 26:111.

57. Cohick WS, Clemmons DR. The insulin-like growth factors. Annu Rev Physiol 1993; 55:131.

58. Nikkhah G, Odin P, Smits A, et al. Platelet-derived growth factor promotes survival of rat and human mesencephalic dopaminergic neurons in culture. Exp Brain Res 1993; 92:516.

59. Alexi T, Hefti F. Trophic actions of transforming growth factor a on mesencephalic dopaminergic neurons developing in culture. Neuroscience 1993; 55:903.

60. Knusel B, Winslow J, Rosenthal A, et al. Promotion of central cholinergic and dopaminergic neuron differentiation by brain-derived neurotrophic factor but not neurotrophin 3. Proc Natl Acad Sci USA 1991; 88:961.

61. Beck KD, Knusel B, Hefti F. The nature of the trophic action of brain-derived neurotrophic factor, des (1-3)insulin-like growth factor, and basic fibroblast growth factor on mesencephalic dopaminergic neurons developing in culture. Neuroscience 1993; 52:855.

62. Hyman C, Hofer M, Barde YA, etal.BDNFisaneurotrophic factor for dopaminergic neurons of the substantia nigra. Nature 1991; 350:230.

63. Hyman C, Squinto SP, Juhasz M, Spina MB, Cedarbaum JM, Furth ME, Lindsay RM. Brain-derived neurotrophic factor (BDNF) protects nigral dopamine neurons from 6hidroxydopamine and MPP+neurotoxicity. Neurology 1991; 41(supl 1):324.

64. Spina MB, Squinto SP, Miller J. Brain-derived neurotrophic factor protects dopamine neurons against 6-hydroxydopamine and N-methyl-4-phenylpyridinium ion toxicity: involvement of the glutathione system. J Neurochem 1992; 59:99.

65. Sauer H, Fischer W, Nikkhah G, et al. Brain-derived neurotrophic factor enhances function rather than survival of intrastriatal dopamine cell-rich grafts. Brain Res 1993; 626:37.

66. Hofer M, Pagliusi SR, Honn A, et al. Regional distribution of brain-derived neurotrophic factor mRNA in the adult mouse brain. EMBO J 1990; 9:2459.

67. Bean AJ, Elde RE, Cao Y, et al. Expression of acidic and basic fibroblast growth factors in the substantia nigra of rat, monkey, and human. Proc Natl Acad Sci USA $1991 ; 88: 10237$.
68. Maisonpierre PC, Belluscio L, Friedman B. NT-3,BDNF and NGF in the developing rat nervous system: parallel as well as reciprocal patterns of expression. Neuron 1990; 5:501.

69. Gall CM, Gold SJ, Isackson PJ, and Seroogy KB. Brain-derived neurotrophin-3 mRNAs are expressed in ventral midbrain regions containing dopaminergic neurons. Mol Cell Neurosci 1992; 3:56.

70. Levvi-Montalcini R. The nerve growth factor 35 years later. Science 1987; 237:1154.

71. Levi A, Alemá S. The mechanism of action of nerve growth factor. Annu Rev Pharmacol Toxicol 1991;31:205.

72. Hartikka J, Hefti F. Development of septal cholinergic neurons in culture: plating density and glial cells modulate effects of NGF on survival, fiber growth, and expression of transmitter-specific enzymes. J Neurosci 1988; 8:2.967.

73. Large TH, Bodary SC, Clegg DO, et al. Nerve growth factorgene expression in the developing rat brain. Science. 1986; 234:353.

74. Von Bartheld CS, Heuer JF, Bothwell M. Expression of nerve growth factor (NGF) receptors in the brain and retina of chick embryos; comparison with cholinergic development. J Comp Neurol 1991; 310:103.

75. Rakic P. Neuronal-glial interaction during brain development. Trends Neurosci 1981; 4:184.

76. Gadisseux JF, Evrarol P. Glial-neuronal relationship in the developing central nervous system. A histochemicalelectron microscope study of radial glial cell particulate glucogen in normal and reeler mice and the human fetus. Dev Neurosci 1985; 7:12.

77. Di Porzio U, Daguet MC, Glowinski J, Prochiantz A. Effect of striatal cells of in vitro maturation of mesencephalic dopaminergic neurons grown in serum-free conditions. Nature 1980; 288:370.

78. Denis-Donini S, Glowinski J, Prochiantz A. Glial heterogeneity may define the three- dimensional shape of mouse mesencephalic dopaminergic neurons. Nature $1984 ; 307: 641$.

79. Beyer C, Pilgrim C, Reisert I, et al. Cells from embryonic rat striatum cocultured with mesencephalic glia express dopaminergic phenotypes. Neurosci Lett 1991; 128:1.

80. Sladek JR, Marshall D. Nerve-cell grafting in Parkinson's disease. J Neurosurg 1988; 68:337.

81. David S, Aguayo AJ. Axonal elongation into peripheral nervous system «bridges» after central nervous system injury in adult rats. Science $1981 ; 214: 931$.

82. Nutt JG. Parkinsonism: candidate disorder for implanted pumps? Ann N Y Acad Sci 1988; 531:194. 